\title{
THE COSMOLOGICAL TIME FUNCTIONS AND LIGHTLIKE RAYS
}

\author{
FATEMEH KOOHESTANI, NEDA EBRAHIMI, MEHDI VATANDOOST, AND YOUSEF BAHRAMPOUR \\ 1,2,3 Department of Pure Mathematics, Faculty of Mathematics and Computer, Shahid Bahonar \\ University of Kerman, Kerman, Iran, \\ ${ }^{4}$ Department of Mathematics and Computer Sciences, Hakim Sabzevari University.
}

\begin{abstract}
It is proved that all discontinuity points of a finite cosmological time function, $\tau$, are on past lightlike rays. As a result, it is proved that if $(M, g)$ is a chronological space-time without past lightlike rays then there is a representation of $g$ such that its cosmological time function is regular. In addition, by reducing conditions of regularity sufficient conditions for causal simplicity and causal pseudoconvexity of space-time is given. It is also proved that the second condition of regularity can be reduced to satisfies only on inextendible past-directed causal rays if $(M, g)$ be a space-time, conformal with an open subspace of Minkowski space-time or $\tau$ be continuous.
\end{abstract}

PACS: 04.20.-q.

Keywords:space-time, Globally hyperbolic, Cosmological time function, Lorentzian metric.

\section{INTRODUCTION}

The concept of cosmological time function was defined in [1. Time functions are important in study of the global causal theory of space-time. They can be defined arbitrary and may have little physical significance. But the cosmological time function is defined canonically and consequently, study of it gives us important information about space-time. Let us recall its definition.

Let $(M, g)$ be a space-time and $d: M \times M \rightarrow[0, \infty]$ be the Lorentzian distance function. The cosmological time function $\tau: M \rightarrow[0, \infty)$ is defined by:

$$
\tau(q):=\sup _{p \leq q} d(p, q),
$$

where $p \leq q$ means that $q \in J^{+}(p)$.

A time function on the space-time $(M, g)$ in the usual sense is a real valued continuous function which is strictly increasing on causal curves. The existence of such a function on $M$ requires causal stability (no closed causal curve in any Lorentzian metric sufficiently near the space-time metric exists). Although $\tau$ is not always well behaved, if it is regular then it is a Cauchy time function [1]. 
Definition 1. [1] The cosmological time function $\tau$ of $(M, g)$ is regular if and only if:

- $\tau(q)<\infty$, for all $q \in M$,

- $\tau \rightarrow 0$ along every past inextendible causal curve.

The first condition is an assertion that for each point $q$ of the space-time any particle that passes through $q$ has been in existence for a finite time (the space-time has an initial singularity in the strong sense). The second condition asserts that every particle came into existence at the initial singularity.

it is proved [1] that if the cosmological time function of $(M, g)$ is regular then:

- it is an almost every where differentiable time function;

- $(M, g)$ is globally hyperbolic;

- For each $q \in M$ there is a future-directed timelike ray $\gamma_{q}:(0, \tau(q)] \rightarrow M$ that realizes the distance from the initial singularity to $q$, that is $\gamma_{q}$ is future-directed timelike unit geodesic which is maximal on each segment, such that:

$$
\gamma_{q}(\tau(q))=q, \tau\left(\gamma_{q}(t)\right)=t, \text { for } t \in(0, \tau(q)] .
$$

We recall that since the Lorentzian distance is not conformally invariant, $\tau$ is not too and even if $(M, g)$ is globally hyperbolic, $\tau$ is not necessarily regular. But it is proved in 4 that if $(M, g)$ is globally hyperbolic then there is a smooth real function $\Omega>0$ such that $\tau$ is regular, on $(M, \Omega g)$. This gives a characterization for global hyperbolicity.

It is also proved [14] that the regular $\tau$ is differentiable at $q$ iff there exists only one timelike ray of the form (1).

In the whole of this paper we suppose that $\tau$ is finite. We reduce the second condition of regularity and investigate its effects on the cosmological time function and causal properties of space-time.

In the second section, we recall some definitions and theorems which are used widely in this paper. In the third section of this paper it is proved that a point of discontinuity appears on a past lightlike ray. Hence $\tau$ is a time function in space-times with no past lightlike rays. Using this time function it can be shown by a simple proof that non-totally vicious space-times without lightlike rays are globally hyperbolic. A different proof was given in [10].

In addition, it can be asked that is it possible to reduce the second condition in the following way: $\tau \rightarrow 0$, along every past inextendible causal geodesic.

The following example shows that this is not the case in general.

Example 1. 1] Let $M:=\left\{(x, y, t) \in S^{1} \times \mathbb{R} \times \mathbb{R}: t>-1\right\}$ with the metric 


$$
g:=d y^{2}+e^{2 y}\left(d x d t+\left(|t|^{2 \alpha}+\left(e^{y^{2}}-1\right)\right) d x^{2}\right)
$$

Although $\tau$ is going to zero along past inextendible causal geodesics it does not go to zero along causal curves.

In section 4 , we investigate some conditions on $(M, g)$ and $\tau$ which imply that $\tau$ is regular in this situation. In addition, Using the cosmological time function sufficient conditions for causal simplicity and causal pseudoconvexity of space-time is given.

\section{PRELIMINARIES}

The standard notations from Lorentzian geometry are used in this paper. The reader is referred to [2, 11]. We denote with $(M, g)$ a $C^{\infty}$ space-time (a connected, Hausdorff, time orientted Lorentzian manifold) of dimension $n \geq 2$ and signature $(-,+, \ldots,+)$. If $p, q \in M$, then $q \in I^{+}(p)$ (resp. $\left.q \in J^{+}(p)\right)$ means that there is a future directed timelike (resp. causal) curve from $p$ to $q$. $I^{+}(p)$ is called the chronological future and $J^{+}(p)$ the causal future of $p$. Likewise $I^{-}(p)$ and $J^{-}(p)$ are defined and are called chronological and causal past of $p .(M, g)$ is causal (resp. chronological) if there is no closed causal (resp. timelike) curve in it. A causal space-time is globally hyperbolic if $J^{+}(p) \cap J^{-}(q)$ be compact, for every $p, q \in M$ and is causally simple if $J^{ \pm}(p)$ be closed, for every $p \in M$. In addition, a space-time is strongly causal if every point of it has arbitrary small causally convex neighbourhoods. The interested reader is referred to [8] for more details.

If $q \in J^{+}(p)$ the Lorentzian distance $d(p, q)$ is the supremum of the length of all causal curves from $p$ to $q$ and if $q \notin J^{+}(p)$ then $d(p, q)=0$. If $(M, g)$ is strongly causal then there is a conformal class of $g$ such that $d$ and $\tau$ are bounded.

Lemma 1. 6] Let $h$ be an auxiliary complete Riemannian metric on $M$ and let $\rho$ be the associated distance. Let $q \in M$ and $B_{n}(q)=\{r: \rho(q, r)<n\}$ be the open ball of radius $n$ centered at $q$. If $(M, g)$ is strongly causal, then there is a smooth function $\Omega>0$, such that $\operatorname{diam}(M, \Omega g)=$ $\sup \{d(p, q), p, q \in M\}$ is finite and for every $\epsilon>0$ there is $n \in N$ such that if $\gamma: I \rightarrow M$ is any $C^{1}$ causal curve,

$$
\int_{I \cap \gamma^{-1}\left(M-\overline{\left.B_{n}(q)\right)}\right.} \sqrt{-g(\dot{\gamma}, \dot{\gamma})} d t<\epsilon
$$

that is, its many connected pieces contained in the open set $M-\overline{B_{n}(q)}$ have a total Lorentzian length less than $\epsilon$. 
The limit curve theorem will be used several times. The reader is referred to [9] for a strong formulation. All the curves are parametrized by $h$ - arc length, where $h$ is a complete Riemannian metric. A past (future) ray in $(M, g)$ is a maximal past (future) inextendible causal geodesic $\gamma:[0, \infty) \rightarrow M$.

A sequence of causal curves $\gamma_{n}:\left[a_{n}, b_{n}\right] \rightarrow M$ is called limit maximizing if:

$$
L\left(\gamma_{n}\right) \geq d\left(\gamma_{n}\left(a_{n}\right), \gamma_{n}\left(b_{n}\right)\right)-\epsilon_{n}
$$

where $\epsilon_{n} \rightarrow 0$.

The following lemma is used in section 3 and 4 .

Lemma 2. 5. Let $z_{n}$ be a sequence in $M$ with $z_{n} \rightarrow z$. Let $z_{n} \in I^{+}\left(p_{n}\right)$ with finite $d\left(z_{n}, p_{n}\right)$. Let $\gamma_{n}:\left[0, a_{n}\right] \rightarrow M$ be a limit maximizing sequence of causal curves with $\gamma_{n}(0)=z_{n}$ and $\gamma_{n}\left(a_{n}\right)=p_{n}$. Let $\widehat{\gamma_{n}}:[0, \infty) \rightarrow M$ be any future extension of $\gamma_{n}$. Suppose either:

- $p_{n} \rightarrow \infty$, i.e. no subsequent is convergent,

- $d\left(z_{n}, p_{n}\right) \rightarrow \infty$.

Then any limit curve $\gamma:[0, \infty) \rightarrow M$ of the sequence $\widehat{\gamma_{n}}$ is a causal ray starting at $z$.

\section{Continuity of Cosmological time Function And Lightlike Rays}

The cosmological time function $\tau$ is not a time function in general, but if $\tau<\infty$ it has the following property:

$$
q \in J^{+}(p) \Rightarrow \tau(p)+d(p, q) \leq \tau(q)
$$

This implies that $\tau$ is isotone.

Definition 2. A function $t: M \rightarrow R$ which satisfies $q \in J^{+}(p) \Rightarrow t(p) \leq t(q)$ is said to be isotone.

It is proved in $[8]$ that isotones are almost everywhere continuous and differentiable.

Theorem 1. Every isotone function $f: M \rightarrow R$ on $(M, g)$ is almost everywhere continuous and almost everywhere differentiable. Moreover, it is differentiable at $p \in M$ iff it is Gâteauxdifferentiable at $p$. Finally, if $x: I \rightarrow M$ is a timelike curve, the isotone function $f$ is upper/lower semi-continuous at $x_{0}=x\left(t_{0}\right)$ iff $f \circ x$ has the same property at $t_{0}$.

Hence the cosmological time function is almost everywhere continuous. The question is where a point of discontinuity appears. 
Theorem 2. Let $(M, g)$ be a space-time with finite cosmological time function $\tau$. If $\tau$ be discontinuous at $q \in M$ then it lies on a past lightlike ray.

Proof. Since $p \mapsto d(p, q)$, for every $q \in M$, is lower semi-continuous, $\tau$ is lower semi-continuous too. Hence it is not upper semi-continuous at $q$. Consequently, there is a sequence $\left\{q_{n}\right\}$ and $\epsilon>0$ that $q_{n} \rightarrow q$ and $\tau\left(q_{n}\right) \geq \tau(q)+\epsilon$. Suppose that $\left\{p_{n}\right\}$ be a sequence in $M$ such that $d\left(p_{n}, q_{n}\right) \geq \tau\left(q_{n}\right)-1 / n$. (2) implies that $\tau\left(p_{n}\right)+d\left(p_{n}, q_{n}\right) \leq \tau\left(q_{n}\right)$. Since $\tau$ is finite we have $d\left(p_{n}, q_{n}\right) \leq \tau\left(q_{n}\right)-\tau\left(p_{n}\right)$ and consequently:

$\tau\left(q_{n}\right)-1 / n \leq d\left(p_{n}, q_{n}\right) \leq \tau\left(q_{n}\right)-\tau\left(p_{n}\right)$.

This implies that $\tau\left(p_{n}\right) \rightarrow 0$. Let $\left\{\gamma_{n}\right\}$ be a limit maximizing sequence such that for each $n, \gamma_{n}$ : $\left[0, a_{n}\right] \rightarrow M$, is a past-directed timelike curve, $\gamma_{n}(0)=q_{n}, \gamma_{n}\left(a_{n}\right)=p_{n}$, i.e. $L\left(\gamma_{n}\right)>d\left(p_{n}, q_{n}\right)-\epsilon_{n}$ where $\epsilon_{n} \rightarrow 0$.

Let $\widehat{\gamma_{n}}:[0, \infty) \rightarrow M$ be any past inextendible extension of $\gamma_{n}$ and $\gamma:[0, a] \rightarrow M$ be the limit curve of $\widehat{\gamma_{n}}$ with $\gamma(0)=q .\left\{p_{n}\right\}$ diverges to infinity (if $p_{n} \rightarrow p$ then by lower semi continuity of $\tau, \tau(p) \leq \operatorname{limin} f\left(\tau\left(p_{n}\right)\right)$. This implies that $\tau(p)=0$, which is a contradiction). Lemma 2 implies that $\gamma$ is a causal ray. If $\gamma$ is not a null ray, it is timelike. As all the curves are parametrized by arc-length, $\limsup \left(l\left(\left.\gamma_{n}\right|_{[0, b]}\right)\right) \leq l\left(\left.\gamma\right|_{[0, b]}\right)$. There are $\delta, b>0$ such that $l\left(\left.\gamma\right|_{[0, b]}\right)+\delta \leq \epsilon / 2$. Consequently, $l\left(\left.\gamma_{n}\right|_{[0, b]}\right) \leq l\left(\left.\gamma\right|_{[0, b]}\right)+\delta \leq \epsilon / 2$, for suffitiently large $n$. $l\left(\left.\gamma_{n}\right|_{\left[b, a_{n}\right]}\right)=l\left(\gamma_{n}\right)-l\left(\left.\gamma_{n}\right|_{[0, b]}\right) \geq \tau(q)+\epsilon / 2-1 / n-\epsilon_{n}$.

Hence for sufficiently large $n, l\left(\left.\gamma_{n}\right|_{\left[b, a_{n}\right]}\right)>\tau(q)$. Since $\gamma$ is timelike, $\gamma_{n}(b) \in I^{-}(q)$, for suffitiently large $n$. Hence $\tau(q) \geq l\left(\left.\gamma_{n}\right|_{\left[b, a_{n}\right]}\right)>\tau(q)$ which is a contradiction.

As an application of Theorem 2 the following two theorems can be proved.

Theorem 3. Let $(M, g)$ be a space-time without past lightlike rays and $\tau<\infty$ then for each $q$ there is a future-directed unit speed timelike ray $\gamma_{q}:(0, \tau(q)] \rightarrow M$ which is maximal in each segment, such that:

$$
\gamma_{q}(\tau(q))=q, \tau\left(\gamma_{q}(t)\right)=t, t \in(0, \tau(q)]
$$

Proof. Let $q \in M$ and $\left\{p_{n}\right\}$ be a sequence such that $d\left(p_{n}, q\right) \geq \tau(q)-1 / n . p_{n}$ diverges to infinity as it is proved in the pervious theorem. Let $\gamma_{n}:\left[0, a_{n}\right] \rightarrow M, \gamma_{n}(0)=q, \gamma_{n}\left(a_{n}\right)=p_{n}$ be a limit maximizing sequence of curves:

$\epsilon_{n}=d\left(\gamma_{n}\left(a_{n}\right), \gamma_{n}(0)-l\left(\gamma_{n}\left[0, a_{n}\right]\right)\right), \epsilon_{n} \rightarrow 0$.

and $\widehat{\gamma_{n}}$ be a past inextendible extension of $\gamma_{n}$, for $n \in \mathbb{N}$. By using of Lemma 2 and assumption $\widehat{\gamma_{n}}$ converges to a timelike ray $\gamma:[0, \infty) \rightarrow M$. It suffices to prove that, 
$d(\gamma(b), q)=\tau(q)-\tau(\gamma(b))$, for every $b \in[0, \infty)$.

$l\left(\gamma_{n}([0, b])\right)=l\left(\gamma_{n}\right)-l\left(\gamma_{n}\left[b, a_{n}\right]\right) \geq \tau(q)-(1 / n)-\epsilon_{n}-\tau\left(\gamma_{n}(b)\right)$.

Since $\tau$ is continuous we have,

$d(\gamma(b), q) \geq l(\gamma[0, b]) \geq \limsup \left(l\left(\gamma_{n}([0, b])\right)\right) \geq \tau(q)-\limsup \left(\tau\left(\gamma_{n}(b)\right)\right)=\tau(q)-\tau(\gamma(b))$,

and the proof is complete.

The proof of the following theorem is similar to what is given for regular cosmological time functions in [1]

Theorem 4. Let $(M, g)$ be a space-time without past lightlike rays. If $\tau<\infty$ then it is a time function.

Proof. If $q \in I^{+}(p)$ then by using of (2) it is clear that $\tau(p)<\tau(q)$. Assume that $q \in J^{+}(p)-I^{+}(p)$ then there is a lightlike geodesic from $p$ to $q$. Let $\gamma_{p}$ be the timelike ray to $p$ guaranteed by Theorem 3 and $x \in \gamma_{p}$. By cutting the corner argument near $p$ we have:

$d(x, q)>d(x, p)+d(p, q)$. Consequently, $\tau(q)-\tau(p) \geq d(x, q)>d(x, p)=\tau(p)-\tau(x)>0$ and the proof is complete.

The following theorem was proved in 10 .

Theorem 5. Non-totally vicious space-time $(M, g)$ with no lightlike rays is a globally hyperbolic space-time.

It is proved in 10 that a chronological space-time without lightlike lines is stably causal and consequently has a time function. Then using this time function the above theorem is proved. In this paper, by using of Theorem 4 and the following lemmas it can be shown that there is a representation of $(M, g)$ such that the cosmological time function is regular.

Lemma 3. Let $(M, g)$ be a chronological space-time without past (or future) lightlike rays then $(M, g)$ is strongly causal.

Proof. Suppose by contradiction that $(M, g)$ is not strongly causal. Then there is $p \in M$ and a sequence of arbitrary small relatively compact neighbourhoods $U_{n}, n \in \mathbb{N}$, of $p$ which are not causally convex, i.e, for every $U_{n}$ there exist $p_{n}, q_{n} \in U_{n}$ and a causal curve $\gamma_{n}$ from $p_{n}$ to $q_{n}$ which are not contained in $U_{n}, p_{n} \rightarrow p, q_{n} \rightarrow q$ and $p=q$. The second part of the limit curve theorem, Theorem 3.1 [9, implies that one of the following cases occur:

1) $\gamma_{n} \mathrm{~s}$ are contained in a compact set. Since $M$ is chronological, the limit curve $\gamma_{q}$ is a closed maximal lightlike curve. The curve $\gamma$ making infinite rounds around $\gamma_{q}$ is a past lightlike ray which 
is a contradition.

2) $\gamma_{n}$ s are not contained in a compact set. Then $\gamma_{p} \circ \gamma_{q}=\gamma$ is the limit curve of $\gamma_{n}$ s. Since $\gamma$ is not a lightlike line, the chronology violating set is non empty. This is a contradiction since $\tau<\infty$.

Lemma 2 and Lemma 3 imply the following theorem.

Theorem 6. Let $(M, g)$ be a chronological space-time without past lightlike rays then there is a positive real function $\Omega$, that the cosmological time function of $(M, \Omega g)$ is regular.

Proof. $(M, g)$ is strongly causal by using of Lemma 3. Let $q_{0} \in M$ and $\Omega>0$ be given as in Lemma 1. It is clear that $\tau$ is finite. Suppose by contradiction that there is a past inextendible causal curve $\eta:[0, \infty) \rightarrow M, \eta(0)=p$ such that $\tau \rightarrow a>0$, along $\eta$. Choose $n$ such that the length of any causal curve out of $\overline{B_{n}\left(q_{0}\right)}$ is less than $\epsilon<a$ and $p \in B_{n}\left(q_{0}\right)$. Since $M$ is strongly causal it is non-total imprisoning and consequently there is $t_{0} \in \mathbb{R}$ such that $\eta(t) \in M-\overline{B_{n}\left(q_{0}\right)}$, for $t>t_{0}$. In addition suppose that $\left\{t_{n}\right\}, t_{n} \rightarrow \infty$, be a sequence of real numbers and $p_{n}=\eta\left(t_{n}\right)$. Let $\gamma_{p_{n}}$ be

the maximal timelike ray guaranteed by Theorem 3 . Since $l\left(\gamma_{p_{n}}\right)=\tau\left(p_{n}\right)>\epsilon, \gamma_{p_{n}} \cap \overline{B_{n}\left(q_{0}\right)} \neq \emptyset$. Again by using of non-total imprisoning condition, $\gamma_{p_{n}}$ escape $\overline{B_{n}\left(q_{0}\right)}$ in a point $q_{n}=\gamma_{p_{n}}\left(s_{n}\right)$. Since $\overline{B_{n}\left(q_{0}\right)}$ is compact $q_{n} \rightarrow q$. let $\gamma_{n}^{\prime}$ be a reparametrization of $\gamma_{p_{n}} \mid\left[0, s_{n}\right]$ with $h$ arc length in such a way that $\gamma_{n}^{\prime}(t)=\gamma_{p_{n}}\left(s_{n}-t\right) . \gamma_{n}^{\prime} \rightarrow \gamma, \gamma(0)=q$.

Since $\gamma_{p_{n}}$ is maximal, for every $n$, Lemma 2 implies that $\gamma$ is a timelike ray.

Indeed, we have:

$\tau(\gamma(b))-\tau(\gamma(0))=\limsup \left(\tau\left(\gamma_{n}^{\prime}(b)\right)-\tau\left(\gamma_{n}^{\prime}(0)\right)=\limsup \left(l\left(\gamma_{n}^{\prime} \mid[0, b]\right)\right) \leq l(\gamma \mid[0, b])=d(\gamma(0), \gamma(b)) \leq\right.$ $\operatorname{limin} f\left(d\left(\gamma_{n}^{\prime}(0), \gamma_{n}^{\prime}(b)\right)=\tau(\gamma(b))-\tau(\gamma(0))\right.$, Since $\tau$ is continuous. In addition, $\tau$ is a time function and consequently $\tau(\gamma(b))-\tau(\gamma(0))>0$.

Let $\eta_{n}=\gamma_{n}^{\prime} \circ \eta . \gamma$ and $\eta$ are the limit curves of $\eta_{n}$ and non of them are lightlike ray. The limit curve theorem implies that $\eta \subset I^{+}(q)$. This means that $M$ has a TIF, which is a contradiction since $M$ has no past lightlike ray and the boundary of a TIF is generated by past lightlike rays.

A non- totally vicious space-time without lightlike rays is choronological [7] and Theorem 5 is easily given by using of Theorem 6 .

\section{The COSMOLOGICAL TIME FUNCTION AND CAUSAL RAYS}

In this section, we reduce the second condition of regularity and investigate the causality properties of space-times which satisfy:

- $\tau$ is finite;

- $\tau \rightarrow 0$ on past inextendible causal geodesics (or on past null rays). 
Example 1 shows that in this case $\tau$ is not necessarily regular. Indeed, it can be checked that it is not globally hyperbolic. Causal pseudoconvexity and causal simplicity are weaker conditions than global hyperbolicity. We will prove that in a reflective space-time the above conditions imply causal pseudoconvexity (or causal simplicity). The space-time $(M, g)$ is called causally pseudoconvex if for each compact set $K$ there exists a compact set $K^{\prime}$ such that each geodesic with both end points in $K$ has its image in $K^{\prime}$.

Definition 3. 12] Assume $p_{n} \rightarrow p$ and $q_{n} \rightarrow q$ for distinct points $p$ and $q$ in space-time $M$. We say that space-time $M$ has causal limit geodesic segment property (LGS), if each pair $p_{n}$ and $q_{n}$ can be joined by a geodesic segment, then there is a limit geodesic segment from $p$ to $q$.

we can define causal, null or maximally null (LGS) property by restricting the condition of the above definition to causal, null or maximally null geodesics, respectively. Theorem 7 , Theorem 8 and Lemma 4 are used to prove the main results of this section. The following theorem gives a characterization for pseudoconvexity.

Theorem 7. 12] Let $(M, g)$ be a strongly causal space-time. Then it is (null or maximal null) causal pseudoconvex if and only if it has (null or maximally null) causal LGS property.

Lemma 4. If the cosmological time function $(M, g)$ satisfies the following conditions:

- $\tau<\infty$;

- $\tau \rightarrow 0$, along past lightlike rays,

then $(M, g)$ is non-totally imprisoning.

Proof. Since $\tau<\infty,(M, g)$ is chronological. Suppose by contradiction that there is a past inextendible causal curve which is imprisoned in a compact set $K$. Hence there is a lightlike line which is imprisoned in $K$ [7. Since $K$ is compact there are $p_{i} \in M, i=1, \ldots, n$, that $K \subset \cup I^{+}\left(p_{i}\right)$ and consequently $\tau(x) \geq \min \left(\tau\left(p_{i}\right)\right), i=1, \ldots, n$, for all $x \in K$. But it is a contradiction to assumption.

Theorem 8. [11] Let $S \subset M$, and set $B=\partial I^{+}(S)$. Then if $x \in B$, there exists a null geodesic $\eta \subseteq B$ with future endpoint $x$ and which is either past-endless or has a past endpoint on $S$.

Theorem 9. 13] Assume that $(M, g)$ be a causal space-time, but not causally simple. Then there are (1) $p, q \in M$ such that $p$ has a future inextendible maximal null geodesic ray in $I^{-}(p)$ or (2) $q$ has a past inextendible maximal null geodesic ray in $\partial I^{+}(q)$. Conversely, assume $(M, g)$ has a $p$ (resp. q) such that $\partial I^{-}(p)$ has a future null geodesic ray (resp. $\partial I^{+}(p)$ has a past inextendible maximal null geodesic ray), then $(M, g)$ is not causally simple. 
Remark. We recall that $(M, g)$ is reflecting iff $p \in \overline{I^{-}(q)} \Leftrightarrow q \in \overline{I^{+}(p)}$, for $p, q \in M$.

Theorem 10. Let $(M, g)$ be a reflecting space-time such that its cosmological time function $\tau$ has the following properties:

- $\tau$ is finite;

- $\tau \rightarrow 0$, along every past lightlike ray $\gamma$;

then $(M, g)$ is causally simple.

Proof. Lemma 4 implies that $(M, g)$ is causal. Suppose by contradiction that $(M, g)$ is not causally simple. Hence there are $p, q \in M$ such that $q \in \overline{J^{+}(p)}-J^{+}(p)$ and (1) or (2) in Theorem 9 occurs: In the first case there is a past lightlike ray $\gamma$ which is contained $q$ and lies in $\partial I^{+}(p)$. Since $(M, g)$ is reflecting, there is a sequence $\left\{p_{n}\right\}, p_{n} \rightarrow p$, such that $p_{n} \in I^{-}(q)$. Lower semi continuity of cosmological time function implies that $\tau(p) \leq \liminf \tau\left(p_{n}\right) \leq \tau(q)$. $q$ can be choosen arbitrary on the causal ray $\gamma$ and consequently $\tau(\gamma) \geq \tau(p)$ which is a contradiction, since $\tau \rightarrow 0$ along it. Again in the second case reflectivity of $(M, g)$ implies that $q$ lies in $\partial I^{+}(p)$, hence by Theorem 8 there is a past lightlike ray $\alpha$ with end point $q$ in $\partial I^{+}(p)$. Indeed, it can not be a null geodesic from $p$ to $q$ since $q \notin J^{+}(p)$. The proof in this case is similar to case (1).

Theorem 11. Let $(M, g)$ be a reflective space-time such that:

- $\tau$ is finite,

- $\tau \rightarrow 0$ along past inextendible causal geodesics,

then $(M, g)$ is causally pseudoconvex.

Proof. Theorem 10 implies that $(M, g)$ is causally simple. Suppose by contradiction that $(M, g)$ is not causally pseudoconvex then Theorem 7 implies that there are sequences $\left\{p_{n}\right\},\left\{q_{n}\right\}$ and geodesics $\gamma_{n}$ from $p_{n}$ to $q_{n}$ such that $p_{n} \rightarrow p, q_{n} \rightarrow q$ but there limit curve $\gamma$ with $\gamma(0)=q$ does not contain $p$. Hence limit curve theorem implies that $\gamma(t) \in J^{+}(p)$ and consequently $\tau(p) \leq \tau(\gamma(t))$ which is a contradiction, since $\gamma$ is an inextendible past geodesic.

Theorem 12. Let $(M, g)$ be a space-time which satisfies the following properties:

- $\tau<\infty$;

- $\tau \rightarrow 0$, along past inextendible causal rays;

- $\tau$ is continuous;

then $\tau$ is regular. 
Proof. Suppose by contradiction that $\tau \nrightarrow 0$ along a past inextendible causal curve $\gamma:[0, \infty) \rightarrow M$. Let $p_{n}=\gamma\left(t_{n}\right)$, where $t_{n} \rightarrow \infty$ and $\gamma_{n}:\left[0, a_{n}\right] \rightarrow M, \gamma_{n}(0)=p$ and $\gamma_{n}\left(a_{n}\right)=p_{n}$ be a limit maximizing sequence. Since by Lemma $4(M, g)$ is non-total imprisoning, $\left\{p_{n}\right\}$ escapes to infinity. Consequently, the limit curve of $\gamma_{n}, \eta:[0, \infty) \rightarrow M, \eta(0)=p$ is a causal ray. $\gamma_{n}(t) \in I^{+}\left(p_{m}\right)$, for $m \geq n$. Hence $\eta \subset \overline{I^{+}(\gamma)}$. Since $\tau>a$ on $I^{+}(\gamma)$ and it is continuous, $\tau(\eta(t)) \geq a$, for every $t$, which is a contradiction.

Theorem 13. Let $(M, g)$ be a space-time of dimension $n+1$, conformally embedded as an open subset in Minkowski space-time $\left(E^{n, 1}, h\right)$, and its cosmological time function satisfies the following properties:

- $\tau<\infty$;

- $\tau \rightarrow 0$, along past causal rays;

then $\tau$ is regular.

Proof. Suppose that $\tau \rightarrow a, a \neq 0$, along a past inextendible causal curve $\gamma:[0, \infty) \rightarrow M$. Let $p_{n}, \gamma_{n}$ and $\eta$ be as in the pervious theorem. It is trivial that $\eta \subseteq \overline{I^{+}(\gamma)} . \partial\left(I^{+}(\gamma)\right)$ is an achronal boundary. $\tau>a$ on $I^{+}(\gamma)$, but $\tau \rightarrow 0$ on $\eta$. Hence $\eta$ has to escape $I^{+}(\gamma)$ in a point $x=\eta(s)$ and $\eta \mid[s, \infty) \subseteq \partial I^{+}(\gamma)$. This implies that $\eta$ is not a timelike ray. In addtion, if $\eta^{\prime}(0)=w$ then $<w, \alpha^{\prime}>_{h}=0$, for any lightlike ray $\alpha$ in $\partial I^{+}(\gamma)$, since $M$ is an open subset of Minkowski space-time. Hence $<w, \eta^{\prime}>_{h}=0$, which is a contradiction.

\section{REFERENCES}

[1] Andersson, L., Galloway, G., and Howard, R., "The cosmological time function," Class. Quantum Grav. 15, 309 (1998).

[2] Beem, J. K., Ehrlich, P. E., and Easley, K. L., Global Lorentzian geometry (Marcel Dekker, New york, 1996).

[3] Beem, J. K., and Parker, P., "Pseudoconvexity and Geodesic Connectedness" annali di Matematica pura ed applicate. (1989) 137-142 Vol CLV (IV).

[4] Ebrahimi, N., "Some observations on cosmological time functions," J. Math. Phys. 54, (2013).

[5] Eschenburg, J. H., and Galloway, G. J., "Lines in space-times," Comm. Math. Phys., 148, 209-216 (1992).

[6] Minguzzi, E., "Characterization of some causality conditions through the continuity of Lorentzian distance," J. Geom. Phys. 59, 827 (2009).

[7] Minguzzi, E., "Non-imprisonment conditions on space-time," J. Math. Phys. 49, 062503 (2008).

[8] Minguzzi, E., "Lorentzian causality theory," Living Rev. Relativ. 22, 1, (2019).

[9] Minguzzi, E., "Limit curve theorems in Lorentzian geometry," J. Math. Phys. 49, 092501 (2008).

[10] Minguzzi, E., "Chronological space-times without lightlike lines are stably causal," Commun. Math. Phys. 288, 801-819 (2008). 
[11] Penrose, R., Theqniques of Differential Topology in Relativity ( CBMS-NSF regional conference series in applied mathematics. SIAM, Philadelphia, (1972).

[12] Vatandoost. M., Pourkhandani. R., and Ebrahimi. N., " On null and causal pseudoconvex space-times," J. Math. Phys. 60, 012502 (2019).

[13] Vatandoost. M., Pourkhandani. R., and Ebrahimi. N., " On null and causal pseudoconvex space-times and naked singularities", submitted.

[14] Xiaojun, C., and Liang, Jin., "The negative of regular cosmological time function is a viscosity solution," J. Math. Phy. 55, 102705 (2014).

Email address: koohestanifateme@gmail.com

Email address: n_ebrahimi@uk.ac.ir

Email address: m.vatandoost@hsu.ac.ir

Email address: bahram@uk.ac.ir 